\title{
An unusual presentation of anomalous left coronary artery from the pulmonary artery (ALCAPA) syndrome in a 70-year-old man: a case report
}

Antonio Vizzuso ${ }^{1}$, Riccardo Righi², Michela Zerbini², Stela Gamanji', Paolo Cucchi ${ }^{1}$, Francesco Gallo ${ }^{3}$, Melchiore Giganti ${ }^{1}$, Giorgio Benea ${ }^{2}$ and Aldo Carnevale ${ }^{1 *}$

\begin{abstract}
Background: We present a rare case of anomalous origin of the left coronary artery from the pulmonary artery syndrome in an elderly man, and we describe coronary computed tomographic angiographic imaging findings to improve diagnostic confidence for the evaluation of this uncommon coronary artery anomaly.

Case presentation: A 70-year-old Caucasian man came to our hospital with slight limitation of physical activity (New York Heart Association class II). He was asymptomatic for angina, syncope, and palpitations. Cardiac magnetic resonance imaging was performed after echocardiography because a hypertrophic cardiomyopathy was suspected; a plausible coronary artery anomaly was demonstrated as collateral evidence. Subsequently, coronary computed tomographic angiography showed the anomalous origin of left coronary artery from the pulmonary artery; the coronary vessels appeared markedly dilated and tortuous. Dilated intercoronary vessels along the epicardial surface of the heart and dilated bronchial arteries, corresponding to collateral pathways, were observed. Left ventricular hypertrophy, delayed subendocardial enhancement, and mitral insufficiency were better evaluated on magnetic resonance images. Invasive coronary angiography confirmed the main findings. Given the patient's age and clinical performance, surveillance with medical management was considered appropriate, and surgical repair was avoided.

Conclusions: Confidence with the anatomic pattern and clinical significance of this anomalous condition is necessary to improve cardiac imaging evaluation ability. In our patient, coronary computed tomographic angiography proved to be a reliable imaging approach, superior to invasive coronary angiography in terms of diagnostic performance and patient safety.
\end{abstract}

Keywords: ALCAPA syndrome, Coronary vessel anomalies, Coronary angiography, Computed tomographic angiography, Coronary vessels

\footnotetext{
* Correspondence: aldocarnevale@hotmail.it

'Department of Morphology, Surgery and Experimental Medicine, Section of

Radiology, University of Ferrara, Via Ludovico Ariosto 35, 44121 Ferrara, Italy

Full list of author information is available at the end of the article
}

(c) The Author(s). 2018 Open Access This article is distributed under the terms of the Creative Commons Attribution 4.0 International License (http://creativecommons.org/licenses/by/4.0/), which permits unrestricted use, distribution, and reproduction in any medium, provided you give appropriate credit to the original author(s) and the source, provide a link to the Creative Commons license, and indicate if changes were made. The Creative Commons Public Domain Dedication waiver (http://creativecommons.org/publicdomain/zero/1.0/) applies to the data made available in this article, unless otherwise stated. 


\section{Background}

Anomalous left coronary artery from pulmonary artery (ALCAPA) syndrome (or Bland-White-Garland syndrome) is a rare, hemodynamically significant congenital coronary artery anomaly affecting 1 per 300,000 live births, accounting for $0.25-0.5 \%$ of all congenital cardiac diseases [1]. It is extremely rare for ALCAPA syndrome to be diagnosed in an adult, because the natural history of this malformation mostly leads to death within the first year of life if untreated [2]. The extent of collateral pathway development between the right and left coronary arteries (RCA and LCA, respectively) determines the extent of myocardial ischemia and the gravity of cardiovascular sequelae [3]. The aim of this article is to describe an unusual case of ALCAPA syndrome in a 70-year-old man with mild clinical manifestations.

\section{Case presentation}

A 70-year-old retired Caucasian man, a former electrical society employee, presented to our hospital with moderate dyspnea on exertion (slight limitation of physical activity; New York Heart Association [NYHA] class II). He was asymptomatic for anginal pain, palpitations, or syncope. His past medical history included well-controlled hypertension, inguinal hernioplasty, and right total hip replacement for arthritis secondary to hip dysplasia. His family history revealed an unspecified heart condition in his father, who had died aged 55 years old. He denied smoking, drinking alcohol to excess, and use of recreational drugs. He did not have diabetes or hypercholesterolemia. He was receiving therapy with ramipril.

The patient's vital signs at presentation were blood pressure $140 / 80 \mathrm{mmHg}$, pulse 58 beats/min with regular rhythm, and body temperature $36.0{ }^{\circ} \mathrm{C}$. His body mass index was $30.8 \mathrm{~kg} / \mathrm{m}^{2}$.

He appeared alert, oriented, and cooperative. His lungs were clear to auscultation and percussion bilaterally; no cardiac murmurs were appreciated. His abdomen was globose and nontender to palpation. His bowel sounds were normal in quality and intensity in all areas.

Mild lower extremity pitting edema was noted, but neither cyanosis nor clubbing was present.

Cranial nerves III-XII were intact; the results of motor and sensory examination of the patient's upper and lower extremities were normal. The patient's reflexes were normal and symmetrical bilaterally in both extremities.

The patient's laboratory findings were unremarkable, except for a brain natriuretic peptide value of $1441 \mathrm{pg} / \mathrm{ml}$ (white blood cell [WBC] count $8.08 \times 10^{3} / \mu \mathrm{l}$, neutrophils $6 \times 10^{3} / \mu \mathrm{l}$, red blood cell [RBC] count $4.63 \times 10^{6} / \mu \mathrm{l}$, mean corpuscular volume $88 \mathrm{fl}$, mean corpuscular hemoglobin concentration $32 \mathrm{~g} / \mathrm{dl}$, hemoglobin $13.9 \mathrm{~g} / \mathrm{dl}$, platelets $218 \times 10^{3} / \mu \mathrm{l}$, creatinine $1.11 \mathrm{mg} / \mathrm{dl}$, glomerular filtration rate $65 \mathrm{ml} / \mathrm{min} / \mathrm{m}^{2}$, total protein $6.5 \mathrm{~g} / \mathrm{dl}$, urea $50 \mathrm{mg} / \mathrm{dl}$, $\mathrm{Na}^{+} 142 \mathrm{mmol} / \mathrm{L}, \mathrm{K}^{+} 3.8 \mathrm{mmol} / \mathrm{L}, \mathrm{Cl}^{-} 98 \mathrm{mEq} / \mathrm{L}$, glucose $77 \mathrm{mg} / \mathrm{dl}$, bilirubin $0.58 \mathrm{mg} / \mathrm{dl}$, alkaline phosphatase $67 \mathrm{U} /$ $\mathrm{L}$, alanine aminotransferase $19 \mathrm{U} / \mathrm{L}$; normal urinalysis, with traces of RBCs, WBCs, and bacteria).

Transthoracic echocardiographic examination demonstrated a marked left ventricular concentric hypertrophy, with regular ventricular cavity size. The patient's segmental/global kinetics were preserved, with borderline normal ejection fraction (55\%); an expanded left atrium and a mild mitral regurgitation were noted.

Therefore, cardiac magnetic resonance (CMR) imaging was performed to investigate a suspected hypertrophic cardiomyopathy (Signa HDxt Echospeed 1.5-Tesla magnetic resonance imaging [MRI] scanner; GE Healthcare, Milwaukee, WI, USA). After administration of intravenous contrast material, late gadolinium enhancement (LGE) consistent with chronic subendocardial ischemia was revealed. As collateral evidence, CMR also showed a plausible coronary artery anomaly (Figs. 1 and 2).

Consequently, the patient underwent coronary computed tomographic angiography (CCTA) (Brilliance $\mathrm{iCT}$ 256-slice scanner; Philips Healthcare, Cleveland, $\mathrm{OH}$, USA). A preliminary scan for scoring the amount of coronary calcium was obtained, and the Agatston score was calculated (0). Iodinated contrast media (Iopamiro 370; Bracco Imaging, Milan, Italy) was injected into an antecubital vein.

Nitroglycerin $0.3 \mathrm{mg}$ was sublingually administered immediately before contrast injection. The patient was in sinus rhythm with a heart rate of $75-80$ beats $/ \mathrm{min}$, unvaried after two 5-mg doses of intravenous metoprolol, so retrospective gated CCTA was performed, and reconstruction of cardiac phases from $40 \%$ to $78 \%$ of the $\mathrm{R}-\mathrm{R}$ interval was done.

For proper scan timing, we used a bolus-tracking technique with an ROI placed in the ascending aorta. When the attenuation values in the selected area rose to a preset threshold (150 Hounsfield units), the system automatically played a short, prerecorded breath-hold instruction to the patient, and the scan was automatically commenced.

The following imaging parameters were used for data acquisition: $256 \times 0.625$ detector collimation, 270-ms gantry rotation time, $100-\mathrm{kV}$ tube voltage and $618-\mathrm{mAs}$ tube current-time product, and field of view of $18 \mathrm{~cm}$ with a matrix of $512 \times 512$. The dataset was reconstructed with a model-based iterative reconstruction, and the images were postprocessed and analyzed on an external workstation (Extended Brilliance Workspace; Philips Healthcare).

CCTA imaging clearly revealed that the LCA originated from the pulmonary artery and then bifurcated into the left anterior descending artery and the left 


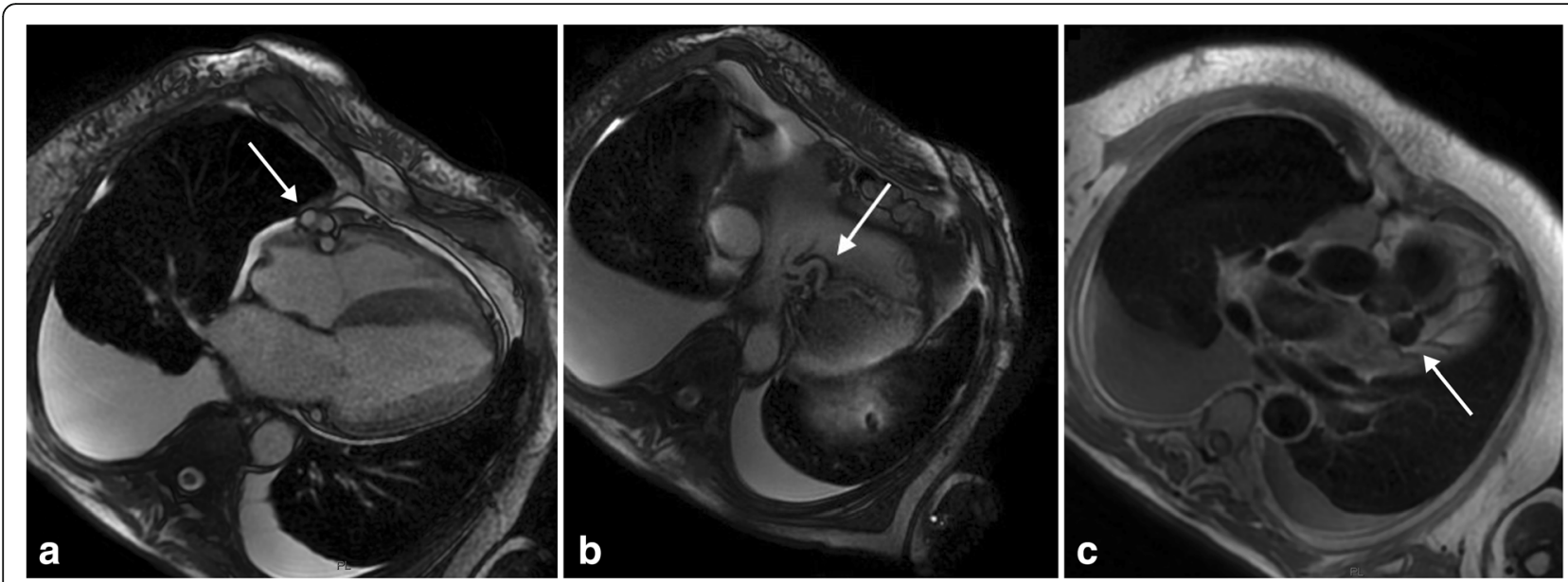

Fig. 1 a and $\mathbf{b}$ Cardiac magnetic resonance imaging steady-state free precession cine sequence, four-chamber plane, showing a plausible coronary artery anomaly: dilated and tortuous right coronary artery (arrow in a), probably anastomosed with the distal portion of the left anterior descending artery (arrow in b). c T1-weighted black-blood sequence demonstrating a left coronary aneurysm (arrow in c), a feature suggestive of a coronary anomaly

circumflex artery. The RCA arose from the right coronary sinus, as normal. The RCA and LCA appeared markedly dilated and tortuous (Figs. 3 and 4). Dilated intercoronary vessels along the epicardial surface of the heart and dilated bronchial arteries corresponded to the collateral pathways of the LCA with the RCA and with systemic vessels, respectively (Fig. 5). The retrograde flow from the anomalous coronary artery to the main pulmonary artery was well depicted (steal phenomenon). The coronary arteries were smooth with no evidence of atherosclerotic disease. Several calcifications at the papillary muscle were observed.

After CCTA, invasive coronary angiography (ICA) was performed. First access was made through the radial artery, then another access was attempted through the femoral vein to confirm the origin of the LCA from the pulmonary artery (Fig. 6).
The risk/benefit ratio for surgery or surveillance with medical treatment was carefully analyzed; owing to the patient's age, signs of mild chronic myocardial ischemia, and only slight limitation of physical activity, a therapy for ischemic cardiomyopathy (including bisoprolol $1.25 \mathrm{mg} / \mathrm{d}$, furosemide $25 \mathrm{mg} / \mathrm{d}$, canrenone $25 \mathrm{mg} / \mathrm{d}$, ramipril $1.25 \mathrm{mg} / \mathrm{d}$, apixaban $5 \mathrm{mg}$ twice daily) was administered, and periodic surveillance was started. In our center, we do not have a standardized follow-up protocol for adult patients with ALCAPA syndrome. In this specific case, our patient underwent a cardiological visit, based on clinical examination and transthoracic echocardiography, 6 months after ICA. The patient was still symptomatic for dyspnea caused by slight physical activity (NYHA class II); an echocardiogram demonstrated stable chamber measures and ejection fraction (left ventricular ejection fraction, 55\%), and mitral regurgitation

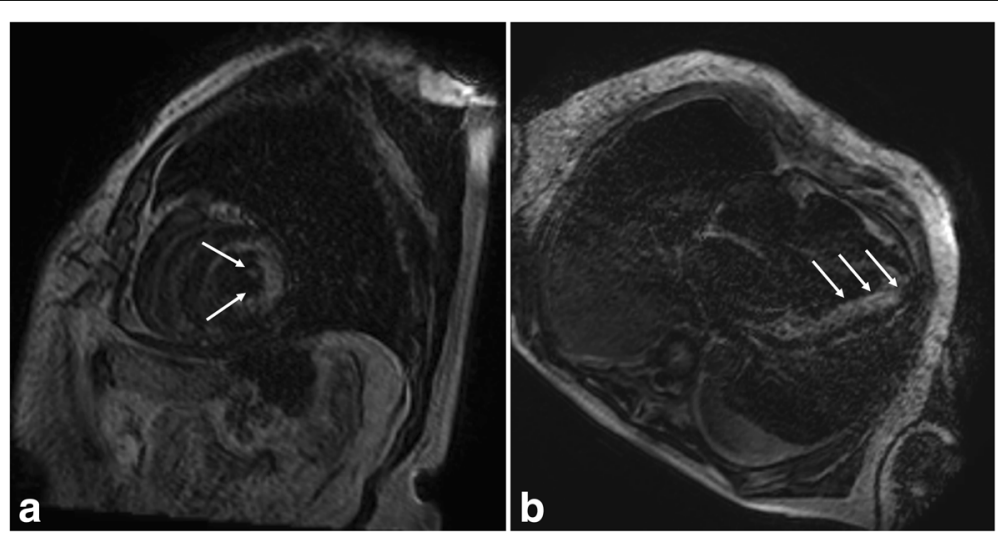

Fig. 2 a and $\mathbf{b}$ Cardiac magnetic resonance imaging short-axis (a) and four-chamber (b) late gadolinium enhancement images showing diffuse subendocardial scar (arrows) at the lateral wall of the left ventricle, a finding consistent with subendocardial infarction subsequent to chronic subendocardial ischemia 


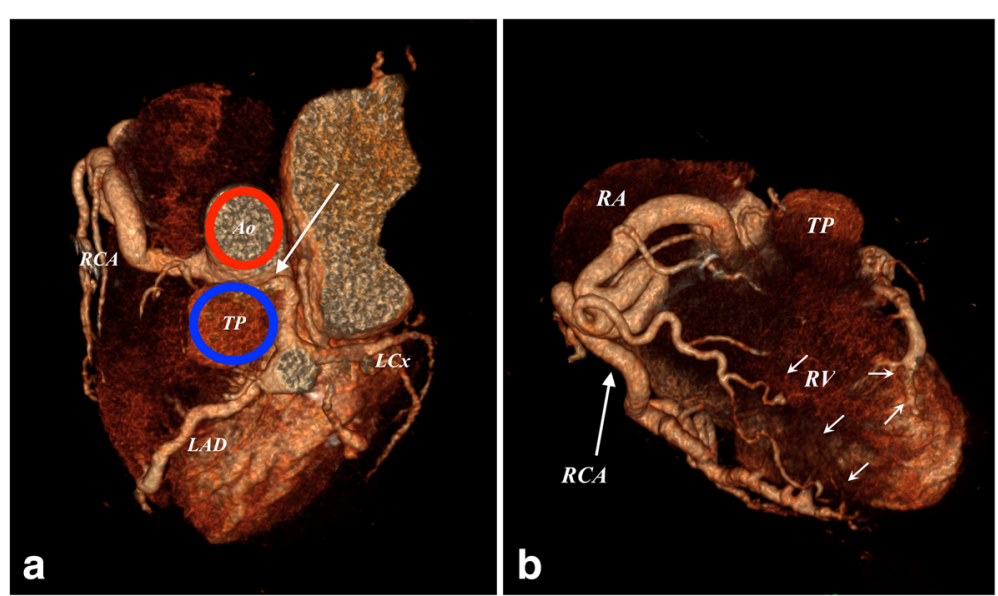

Fig. $3 \mathbf{a}$ and $\mathbf{b}$ Three-dimensional volume-rendered computed tomographic angiograms showing the anomalous origin of the left coronary artery from the pulmonary artery syndrome (arrow in a). Dilated intercoronary arteries (short arrows in $\mathbf{b}$ ) can be seen along the epicardial surface, representing collateral pathways between the RCA and the left coronary artery. Ao Aorta, LAD Left coronary artery, LCx Left circumflex coronary artery, RA Right atrium, RCA Right coronary artery, RV Right ventricle, TP Pulmonary trunk

was still moderate. The estimated pulmonary artery pressure was $55 \mathrm{mmHg}$.

\section{Discussion}

This case report describes a 70-year-old man who presented to our hospital with slight limitation during ordinary activity, and he was diagnosed with ALCAPA syndrome. Owing to the rarity of this condition and its atypical presentation in adulthood, diagnosis can be challenging. Until recently, conventional coronary angiography was the method of choice for detecting coronary anomalies. We present our experience with the diagnosis of ALCAPA syndrome performed by CCTA, highlighting the outstanding features of this disease.
An anomalous origin of a coronary artery from the pulmonary artery is a rare condition, and ALCAPA syndrome represents the most common variant. Other types include anomalous origin of the RCA from the pulmonary artery and anomalous left anterior descending coronary artery or circumflex artery from the pulmonary artery [4]. It is a very rare situation, indeed, when ALCAPA syndrome is diagnosed in an adult, because the natural history of this malformation mostly leads to death within the first year of life if untreated [2].

Two types of ALCAPA syndrome are recognized: an infantile variant and a rarer adult variant $[5,6]$. This anomaly has generally no effect on the hemodynamics of the coronary arteries in the prenatal and early neonatal

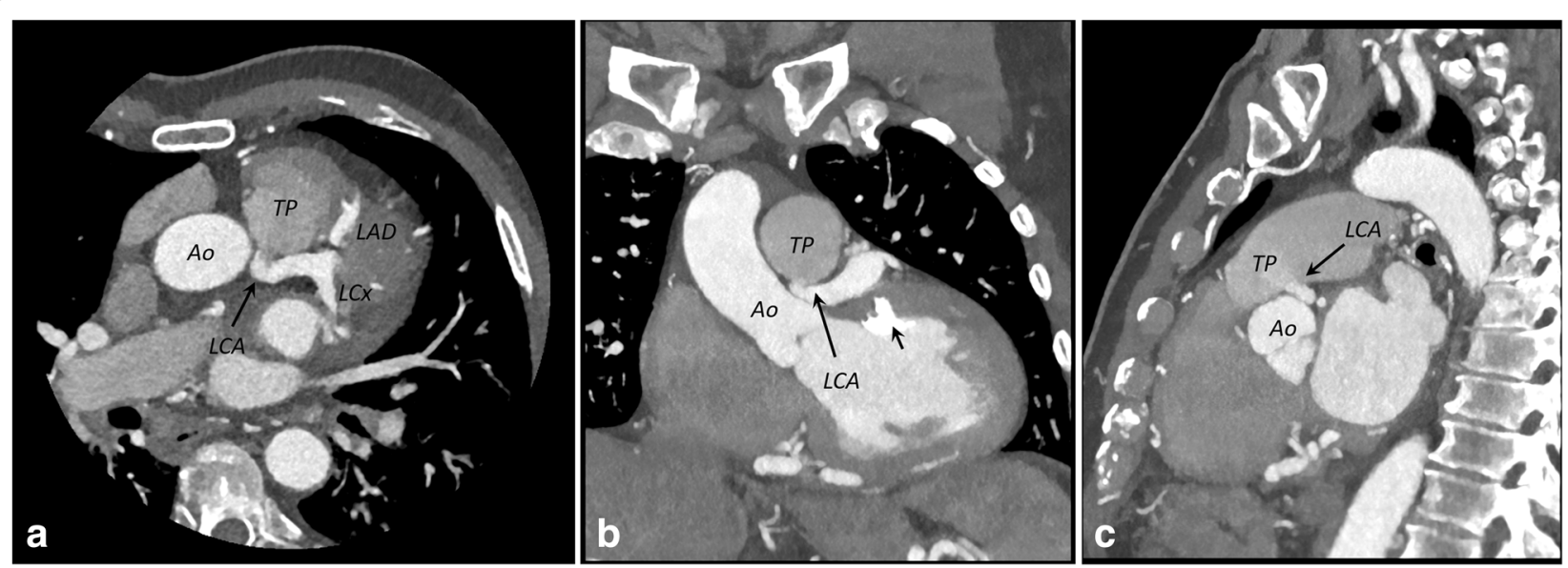

Fig. 4 a-c Axial (a), coronal (b), and sagittal (c) maximum-intensity projection (5 mm) coronary computed tomographic angiographic images depicting the origin of the left coronary artery (LCA) (arrows) from the pulmonary trunk (TP) and not from the Aorta (Ao). Several calcifications are noted at the papillary muscle (short arrow in $\mathbf{b}$ ), resulting from chronic ischemic changes. LAD Left coronary artery, LCX Left circumflex coronary artery 


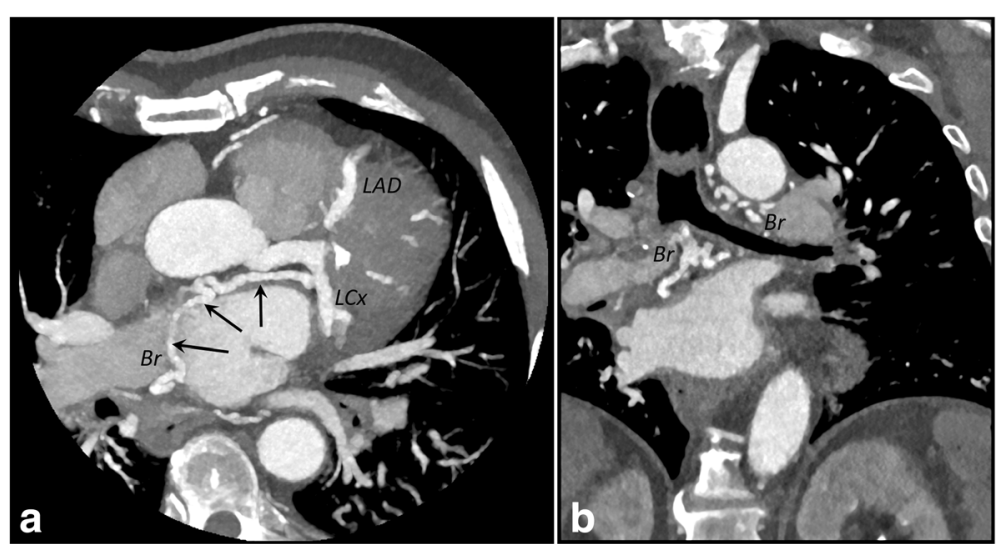

Fig. 5 a and $\mathbf{b}$ Axial (a) and coronal (b) maximum-intensity projection $(10 \mathrm{~mm})$ coronary computed tomographic angiographic images showing dilated bronchial arteries $(\mathrm{Br})$ anastomosing to the left circumflex artery $(\mathrm{LCX})$ (arrows). LAD Left coronary artery

phases, because blood pressure conditions between the systemic circulation and pulmonary arterial circulation are equalized by the patent ductus arteriosus, thus ensuring an antegrade blood flow in the LCA. After birth, the physiological occlusion of the ductus arteriosus lowers the pressure in the pulmonary arterial circulation, causing a decreasing flow rate in the LCA and a conversion of blood flow in the LCA toward the pulmonary artery. In the infantile variant, collaterals do not form between the RCA and LCA, and the retrograde flow in the LCA to the pulmonary artery results in steal of blood from the myocardium, with consequent myocardial ischemia, congestive heart failure, and mitral insufficiency. In infants, ALCAPA syndrome typically manifests with a failure to thrive, profuse sweating, pallor, dyspnea, and atypical chest pain while eating or crying.

The adult type is characterized by the compensatory formation of collaterals between the RCA and the LCA [1]. The extent of collateral circulation between the vessels during the critical period, when pulmonary arterial pressure gradually decreases, determines the extent of myocardial ischemia. The spectrum of clinical manifestations ranges from an asymptomatic course to decompensation of the coronary arterial collateral circulation, with the result of clinical evidence of a previously chronically subclinical ischemia. Ischemic cardiomyopathy, mitral insufficiency, or malignant dysrhythmias causing sudden death may occur secondary to myocardial ischemia $[3,5]$.

Echocardiography aids in diagnosis, notably in pediatric patients and young adults, because it allows the direct visualization of the abnormal origin of the LCA, dilated RCA, retrograde filling of the anomalous coronary artery, abnormal diastolic flow in the pulmonary artery, and abnormal septal or epicardial color flow signals from the collateral vessels $[6,7]$. In our patient, the quality of the echocardiographic images was suboptimal owing to overweight and poor acoustic window: Neither the anomalous ostium of the LCA nor the course of the RCA was noticeable.

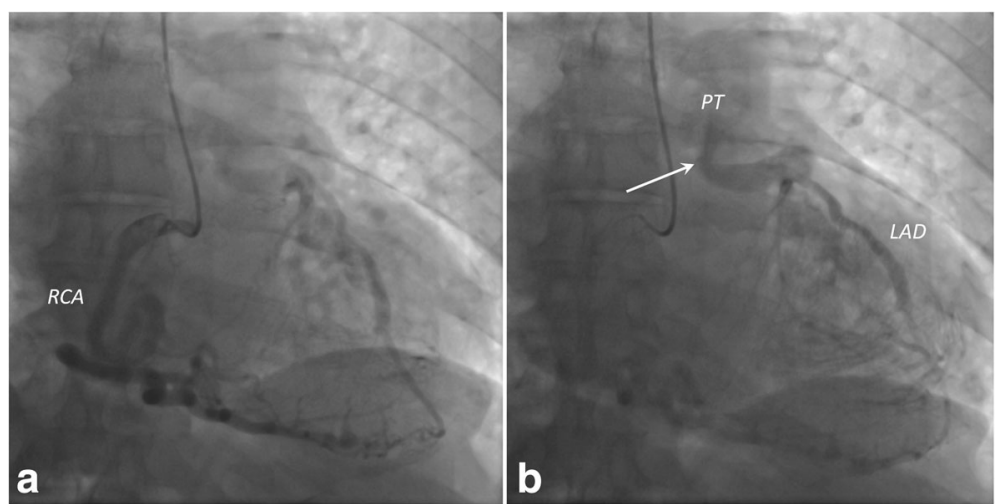

Fig. 6 a Coronary angiogram obtained after right coronary artery (RCA) injection showing tortuous and dilated lumen. b Coronary angiogram demonstrating opacification of both the left anterior descending artery (LAD) and the pulmonary trunk (PT). This finding represents the "steal phenomenon" (arrow), the main diagnostic feature of left coronary artery from the pulmonary artery syndrome 
In recent years, CCTA has emerged as the standard of reference for identification and characterization of coronary artery anomalies. CCTA indeed provides a noninvasive imaging tool to demonstrate the origin and relationship of anomalous arteries to other mediastinal vascular structures, and it enables the use of three-dimensional reformation for delineation of subtle variations in the position and morphology of anomalous vessels [1]; hence, it could be considered the imaging modality of choice to noninvasively delineate coronary vessel anatomy. Moreover, it plays an important role in surgical intervention planning, and it may be a valuable postoperative follow-up tool for adult patients [8]. The short examination time and minimal aftercare make CCTA more practical than ICA [9]. Furthermore, advanced techniques and acquisition protocols, associated with improvements in the reconstruction phase, have enabled progressive dose decrease [10].

CMR imaging is a valuable, noninvasive imaging technique with the ability to assess myocardial viability and function. It can also be used to demonstrate the coronary arteries; however, long examination time and low spatial resolution are the main disadvantages compared with CCTA, limiting its application to demonstrating coronary arteries and their abnormalities [9].

MRI has an advantage over computed tomography (CT) in that it enables concurrent assessment of left ventricular and mitral valve dysfunction as well as signs of chronic myocardial ischemia (namely wall motion abnormalities and LGE) without the use of ionizing radiation. It can also directly demonstrate the reversed flow from the anomalous coronary artery into the pulmonary artery using steady-state free precession cine MRI, and it can help to quantify the degree of left-to-right shunting with phase-contrast imaging. Finally, MRI stress-rest perfusion and LGE imaging techniques can help identify reversible ischemia and subendocardial scarring [3-5]. Delayed subendocardial enhancement seen on MRI scans, a finding caused by subendocardial infarction resulting from chronic subendocardial ischemia, is relevant, especially in asymptomatic adults and those in whom ALCAPA syndrome is incidentally discovered, because it may be predictive of the onset of malignant dysrhythmias. If subendocardial enhancement is seen, surgical repair should be considered [3].

On CCTA imaging, direct visualization of the LCA arising from the main pulmonary artery is the diagnostic hallmark of ALCAPA syndrome. The LCA typically originates from the left inferolateral aspect of the main pulmonary artery just beyond the pulmonary valve. Both the RCA and LCA appear dilated and tortuous in adulthood, with a characteristic "steal phenomenon" (steal of blood flow from the myocardium) well depicted on MRI/CT scans as a retrograde flow from the LCA to the main pulmonary artery. Dilated intercoronary collateral arteries along the epicardial surface of the heart or within the interventricular septum and dilated bronchial arteries can also be observed, representing the collateral pathways between the RCA and the LCA and the systemic collateral vessels to the LCA. Left ventricular hypertrophy and dilation, left ventricular or most commonly global hypokinesis, subendocardial LGE on MRI studies, mitral insufficiency and prolapse, or several calcifications are findings of chronic ischemic changes $[1,3,4,9]$.

The main differential diagnosis for dilation of the coronary arteries includes vasculitis, such as Kawasaki disease or Takayasu arteritis, coronary artery-coronary sinus fistula, atresia, and atherosclerosis-related coronary artery ectasia [3]. Also, ALCAPA syndrome is among the differential diagnoses for massive cardiomegaly in the newborn period.

Surgical repair is the definitive treatment for ALCAPA syndrome, with the aim being to restore a two-coronary artery circulation system. Surgery is performed to correct subendocardial ischemia, thus improving left ventricular function and reducing the risk of malignant arrhythmia and sudden death [4]. Surgery is beneficial for pediatric patients with ALCAPA syndrome because it improves the survival rate; when contemplated for an elderly patient, one must consider unnecessary surgical risk without any clear improvement in the clinical outcome. It may be considered more prudent to medically treat these patients [6], as in our patient's case.

Several surgical approaches are described in the literature. Coronary button transfer (or coronary arterial translocation) is the preferred surgical reparation technique for ALCAPA syndrome, especially in neonates, with low mortality rates and an excellent long-term prognosis. In this strategy, the proximal tract of the anomalous coronary artery is mobilized and anastomosed to the aortic root, whereas the pulmonary trunk is repaired with autologous pericardium. Other operative strategies include the Takeuchi repair (the creation of an aortopulmonary window and an intrapulmonary tunnel that baffles the aorta to the ostium of the anomalous LCA) and bypass graft to restore a two-coronary artery system with ligation of proximal anomalous artery [4]. Long-term follow-up imaging is recommended to evaluate for postsurgical complications.

\section{Conclusions}

Coronary artery anomalies include a wide spectrum of variants with diverse cardiovascular manifestations. Familiarity with the anatomic pattern and clinical significance of each anomaly is necessary to categorize each condition and understand its importance in terms of prognosis. With the increasing use of modern imaging techniques such as CCTA and MRI, many asymptomatic 
patients are now diagnosed incidentally, as in our patient's case. In particular, ALCAPA syndrome is a rare condition, even more unusual if encountered in an elderly patient.

\section{Abbreviations}

ALCAPA: Anomalous left coronary artery from pulmonary artery; CCTA: Coronary computed tomographic angiography; CMR: Cardiac magnetic resonance; CT: Computed tomography; ICA: Invasive coronary angiography; LCA: Left coronary artery; LGE: Late gadolinium enhancement; MRI: Magnetic resonance imaging; NYHA: New York Heart Association; RBC: Red blood cell; RCA: Right coronary artery; WBC: White blood cell

\section{Availability of data and materials}

Data sharing is not applicable to this article, because no datasets were generated or analyzed during the current study.

\section{Authors' contributions}

AV and PC wrote the manuscript with support from AC, who contributed to the final version of the article. MZ, RR, and SG obtained imaging studies and imaging interpretation. FG provided clinical and cardiological assessment. MG and GB supervised the project. All authors read and approved the final manuscript.

\section{Ethics approval and consent to participate}

This case report does not involve any active intervention for patients, and therefore ethics approval is waived.

\section{Consent for publication}

Written informed consent was obtained from the patient for publication of this case report and any accompanying images. A copy of the written consent is available for review by the Editor-in-Chief of this journal.

\section{Competing interests}

The authors declare that they have no competing interests.

\section{Publisher's Note}

Springer Nature remains neutral with regard to jurisdictional claims in published maps and institutional affiliations.

\section{Author details}

${ }^{1}$ Department of Morphology, Surgery and Experimental Medicine, Section of Radiology, University of Ferrara, Via Ludovico Ariosto 35, 44121 Ferrara, Italy. ${ }^{2}$ Department of Interventional and Diagnostic Radiology, Arcispedale Sant'Anna, Via Aldo Moro 8, 44124 Ferrara, Italy. ${ }^{3}$ Department of Medical Science, Cardiovascular Institute, University of Ferrara, Via Ludovico Ariosto 35, 44121 Ferrara, Italy.

Received: 29 May 2018 Accepted: 18 September 2018

Published online: 22 October 2018

\section{References}

1. Shriki JE, Shinbane JS, Rashid MA, Hindoyan A, Withey JG, DeFrance A, et al. Identifying, characterizing, and classifying congenital anomalies of the coronary arteries. Radiographics. 2012;32:453-68. https://doi.org/10.1148/ rg.322115097

2. Uruski $P$, Lipski $D$, Trojnarska $O$, Juszkat $R$, Tykarski $A$. ALCAPA syndrome in a 56-year-old woman with dyspnoea on exertion. Kardiol Pol. 2014;72:1165.

3. Peña $\mathrm{E}$, Nguyen ET, Merchant N, Dennie G. ALCAPA syndrome: not just a pediatric disease. Radiographics. 2009;29:553-65.

4. Agarwal PP, Dennie C, Pena E, Nguyen E, LaBounty T, Yang B, et al. Anomalous coronary arteries that need intervention: review of pre- and postoperative imaging appearances. Radiographics. 2017;37:740-57. https:// doi.org/10.1148/rg.2017160124

5. Heermann P, Heindel W, Schülke C. Coronary artery anomalies: diagnosis and classification based on cardiac CT and MRI (CMR) - from ALCAPA to anomalies of termination. Rofo. 2017;189:29-38.

6. Yew KL, Kang Z, Anum A. Late presentation of ALCAPA syndrome in an elderly Asian lady. Med J Malaysia. 2016;71:217-9.
7. Kothari J, Lakhia K, Solanki P, Parmar D, Boraniya H, Patel S. Anomalous origin of the left coronary artery from the pulmonary artery in adulthood: challenges and outcomes. Korean J Thorac Cardiovasc Surg. 2016;49:383-6. https://doi.org/10.5090/kjtcs.2016.49.5.383

8. di Cesare E, Carbone I, Carriero A, Centonze M, De Cobelli F, De Rosa R, et al. Clinical indications for cardiac computed tomography. From the Working Group of the Cardiac Radiology Section of the Italian Society of Medical Radiology (SIRM). Radiol Med. 2012;117:901-38. https://doi.org/10. 1007/s11547-012-0814-x

9. Al Umairi RS, Al Kindi F, Al Busaidi F. Anomalous origin of the left coronary artery from the pulmonary artery: the role of multislice computed tomography (MSCT). Oman Med J. 2016;31:387-9. https:/doi.org/10.5001/omj.2016.77

10. Maffei E, Martini C, De Crescenzo S, Arcadi T, Clemente A, Capuano E, et al. Low dose CT of the heart: a quantum leap into a new era of cardiovascular imaging. Radiol Med. 2010;115:1179-207. https://doi.org/ 10.1007/s11547-010-0566-4

\section{Ready to submit your research? Choose BMC and benefit from:}

- fast, convenient online submission

- thorough peer review by experienced researchers in your field

- rapid publication on acceptance

- support for research data, including large and complex data types

- gold Open Access which fosters wider collaboration and increased citations

- maximum visibility for your research: over $100 \mathrm{M}$ website views per year

At BMC, research is always in progress.

Learn more biomedcentral.com/submissions 